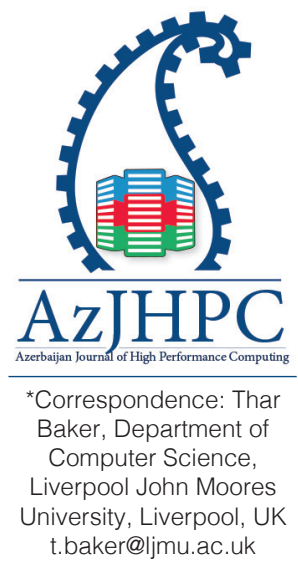

\title{
A Survey of Resource Management Challenges in Multi-cloud Environment: Taxonomy and Empirical Analysis
}

\author{
Bandar Aldawsari ${ }^{1}$, Thar Baker ${ }^{1}$, Muhammad Asim², Zakaria Maamar ${ }^{3}$, \\ Dhiya Al-Jumeily ${ }^{1}$, Mohammed Alkhafajiy ${ }^{1}$ \\ ${ }^{1}$ Department of Computer Science, Liverpool John Moores University, Liverpool, \\ UKB.M.Aldawsari@2012.ljmu.ac.uk; t.baker @ljmu.ac.uk; D.aljumeily@ljmu.ac.uk; \\ M.D.Alkhafajiy@2016.ljmu.ac.uk \\ ${ }^{2}$ Department of Computer Science, National University of Computer and Emerging Sciences, \\ Islamabad, Pakistan, muhammad.asim@nu.edu.pk \\ ${ }^{3}$ College of Technological Innovation, Zayed University, Abu Dhabi, UAE, Zakaria.Maamar@zu.ac.ae
}

\section{Abstract}

Cloud computing has seen a great deal of interest by researchers and industrial firms since its first coined. Different perspectives and research problems, such as energy efficiency, security and threats, to name but a few, have been dealt with and addressed from cloud computing perspective. However, cloud computing environment still encounters a major challenge of how to allocate and manage computational resources efficiently. Furthermore, due to the different architectures and cloud computing networks and models used (i.e., federated clouds, VM migrations, cloud brokerage), the complexity of resource management in the cloud has been increased dramatically. Cloud providers and service consumers have the cloud brokers working as the intermediaries between them, and the confusion among the cloud computing parties (consumers, brokers, data centres and service providers) on who is responsible for managing the request of cloud resources is a key issue. In a traditional scenario, upon renting the various cloud resources from the providers, the cloud brokers engage in subletting and managing these resources to the service consumers. However, providers' usually deal with many brokers, and vice versa, and any dispute of any kind between the providers and the brokers will lead to service unavailability, in which the consumer is the only victim. Therefore, managing cloud resources and services still needs a lot of attention and effort. This paper expresses the survey on the systems of the cloud brokerage resource management issues in multi-cloud environments.

\section{Keywords: Cloud computing}

\section{Introduction}

Cloud computing has witnessed the increase in delivering computing resources as a utility as per cloud consumers dynamically changing needs [1]. Essentially, the purpose is to transfer the responsibility of resource management and allocation 
to the cloud providers, to create demands for the computing resources, to reduce the service launch-time, and to remove the costs of establishing the infrastructure. Basically, the examples of cloud providers include Microsoft Windows Azure, IBM cloud, Amazon Web Services, as well as Google Apps. Indeed, the key characteristics that cloud computing systems exhibit include scalability, reliability, and flexibility [2]. The capitalization of these key characteristics has been the focus of research in the recent past that has optimized the issues such as affordability, security, and availability. Notably, the cloud resource providers, service providers and the service consumers are the key components of the cloud computing system [3]. The cloud providers sublet computing resources to service providers so that they could deploy their services on the cloud and offer them to service consumers. Principally, the service providers rely on the Service Level Agreements (SLA) in service deployment to the multiple computing nodes. The SLA is negotiated between the two parties for the availability of the particular system resource while specifying the use. The SLA may be violated by the extreme resource scarcity caused by the high optimism of the deployment scheme that underestimates the required resources. On the other hand, the cloud system operating costs may increase without the increase in revenue due to the inefficient deployment of the resources of the pessimistic scheme that overestimates the required resources. However, the applicability of the deployment schemes is based on the operating conditions and the specific domain of application. Hence, the process of system design encounters limited scope, reliability, and utility [4].

The reason for the decrease in the performance of the application is that the required storage resources, network, and computing configurations were complex. Ideally, the SLA only provides for the aspects of availability of resources for the infrastructure of cloud computing. The quality of service and the application level lack the agreement for their specification for the particular resources to provide services and application. The majority of the cloud providers are capable of addressing the provider requirements of services and application. However, the main challenge is in choosing the best cloud provider and requires optimized customization, integration, and aggression of the necessary cloud resources [5].

The complexity of cloud resource management significantly increased due to the advent of cloud brokerage and cloud federated systems [3]. The cloud broker, that is a third party, has gained popularity recently for helping the service providers in the selection of the appropriate cloud providers as well as deploying the services in federate or single cloud infrastructure. In reality, the role of the intermediary cloud brokers is to help in service deployment in federated cloud infrastructure, negotiate the conditions of the contract, and identify best cloud provider [NIST]. In fact, in other situations, they engage in multiple cloud providers to host service and rent resources from them. Therefore, they overcharge for the cloud resources more than the cost providers receive [6]. Moreover, in cloud computing, cloud brokerage is an IT role that provides value added services for the cloud providers for the benefit of customers. Additionally, it can achieve the business continuity by mitigating the risk of service downtime. Through the cloud brokerage services providers; users can easily identify the right provide and cloud service which meet their demands. 


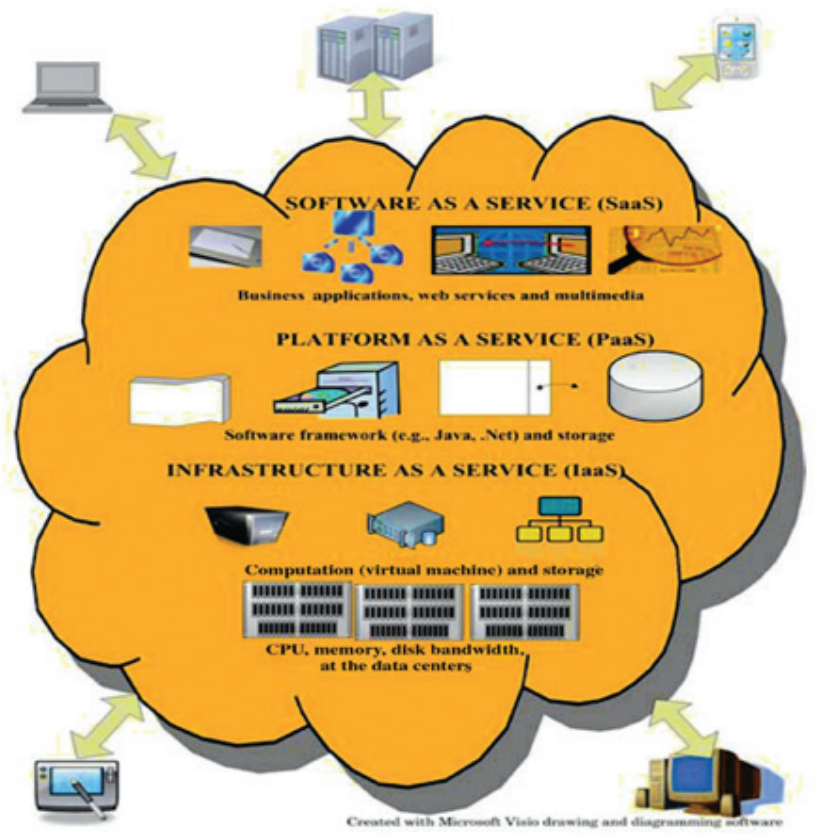

Figure 1 Cloud computing models service

\section{Background}

\subsection{Cloud computing}

According to [9], Cloud computing is the next stage in evolution of the Internet, offering the means through which everything can be delivered to users on demand everywhere at any time from computing power to computing infrastructure, applications, business processes to personal collaboration. Unlike storing information on office computers or servers, cloud computing store data on the Internet, enabling users to access information anywhere using compatible electronic devices. In fact most of these services are accessed from a multitude of servers usually distributed across numerous data centres, and for which the cloud provider is responsible for maintenance [2]. For instance, the word processor application such as Google Docs or Adobe's Buzzword can be used at anytime from anywhere without the need to install them in their local computers. Another common service that cloud computing can offer to its users is storage, e.g., Amazon Web Services, Google App Engine, etc. [7][8].

Cloud computing architecture consists of three layers: application, platform, and infrastructure. As shown in Figure 1, application and platform layers provide Software as a Service (SaaS) and Platform as a Service (PaaS) while infrastructure layer provides Infrastructure/hardware as a Service (laaS). To sum up, cloud computing contains three models of service: Infrastructure as a Service (IaaS), Platform as a Service (PaaS) and Software as a Service (SaaS). 
There are four deployment models of cloud computing: Public clouds, Private clouds, Community clouds and Hybrid clouds [2]. Public clouds provide resources such as storage applications for general use via the cloud provider. These services are either operating on a pay-per-use model or in some cases offered free of cost. Private clouds offer the power, and features of a public cloud, including security, scalability and self-service, but implemented within the corporate firewall. It is managed within the organisation or via a third party. Community clouds provide the opportunity for several organisations to share cloud services so they can form their own community with common concerns. Hybrid clouds are a combination of two or more clouds (community, public or private) to perform distinct functions.

\subsection{Cloud Computing Actors and Characteristics}

The most important factors for cloud computing with significant roles within the cloud environments are listed in Table 1.

According to National Institute of Standards and Terminology (NIST) there are five main characteristics for cloud computing (Table 2):

\subsection{Cloud Broker}

NIST and Gartner have provided a definition of Cloud Service Brokerage [11, 12]. Their definition revolves around a three-pronged categorization. According to the two organisations, cloud broker refers to an entity, which oversees the negotiation of relationships between service providers and cloud providers, and manages the use, performance and delivery of cloud services [13]. Both organisations single out aggregation. The focus of Gartner customisation and NIST intermediation is on promoting the existing service. Gartner integration and NIST arbitration share a similarity that is reflected integration of diverse systems and flexible mediation.

- Aggregation focuses on the delivery of two or multiple services to several

\section{TABLE 1 Could Computing Actors [10]}

\begin{tabular}{|c|l|}
\hline cloud-user & $\begin{array}{l}\text { A person who is authenticated to a cloud-provider but } \\
\text { does not have a financial relationship with the cloud- } \\
\text { provider. }\end{array}$ \\
\hline cloud-provider & $\begin{array}{l}\text { An organization providing network services for which it } \\
\text { changes the cloud-subscribers. A (public) cloud-provider } \\
\text { provides services over the Internet. }\end{array}$ \\
\hline $\begin{array}{c}\text { cloud- } \\
\text { management- } \\
\text { broker }\end{array}$ & $\begin{array}{l}\text { A service providing cloud management capabilities over } \\
\text { and above those of the cloud-provider and/or across } \\
\text { multiple cloud-providers. Service may be implemented as } \\
\text { a commercial service apart from any cloud-provider, as } \\
\text { cross-provider capabilities supplied by a cloud-provider } \\
\text { or as cloud-subscriber-implemented management } \\
\text { capabilities or tools }\end{array}$ \\
\hline unidentified-user & $\begin{array}{l}\text { An entity in the Internet (human or script) that interacts with } \\
\text { a cloud over the network and has not been authenticated. }\end{array}$ \\
\hline
\end{tabular}


customers; however, this does not involve provision new customisation, integration, and functionality, rather it focuses on providing central management of security and SLAs. Service Aggregation models play an important role in the broker systems, they help deploy customer applications across multiple cloud providers [14]. Some jobs across multiple cloud providers' that offer similar or different types of services can be aggregated to meet user requirements. Sometimes, consumers may have specific time and limited budget and need their application to be distributed across multiple cloud providers, which meet their requirements.

- Customisation involves increasing or modifying capacities for improvement and promotion of service, alongside the analytics.

- Integration tackles the problems associated with the functioning of independent services in a combined manner; this usually involves combining processes in a layer/ combining a vertical cloud data or stack. Conventional methods such as orchestration, mediation and transformation offer solutions. Generally, the Integration process, allows the communicated data and information to be integrated via mediation technique. In addition, service discretion plays an essential role in cloud brokerage systems especially in the integration process. It enables the system to discover, deploy, and manage multiple services [15]. In the broker system, users need to know all the related information of services such as capabilities of cloud resources, quality and availability.

\subsection{Cloud Service Broker Architectures}

Cloud brokerage solutions are based on existing SaaS/PaaS/laaS, cloud platform, and virtualisation [2]. According to Fowley et al. [16] there are three architectural patterns may be identified:

- Cloud Management: supports monitoring, providing, deployment, and designing cloud resources, for instance, using management portals; this constitutes an

\section{TABLE 2 Cloud Computing Characteristics [10]}

\begin{tabular}{|c|l|}
\hline $\begin{array}{c}\text { On-demand self- } \\
\text { service }\end{array}$ & $\begin{array}{l}\text { The cloud computing vendors can easily and automatically } \\
\text { get the provision of cloud resources on demand whenever } \\
\text { they are required without the need of human interaction. }\end{array}$ \\
\hline $\begin{array}{c}\text { Broad network } \\
\text { access }\end{array}$ & $\begin{array}{l}\text { Cloud capabilities are available over the network, so users } \\
\text { can access them from anywhere once they have a connected } \\
\text { device, such as mobile phones, laptops and PDAs }\end{array}$ \\
\hline $\begin{array}{c}\text { Resource } \\
\text { pooling }\end{array}$ & $\begin{array}{l}\text { Provider's computing resources such as storage, processing, } \\
\text { memory, network bandwidth, virtual machines and email } \\
\text { services are pooled together to serve multiple users }\end{array}$ \\
\hline Rapid elasticity & $\begin{array}{l}\text { Cloud services can be rapidly and elastically provisioned as } \\
\text { they are unlimited and can be purchased in any quantity at } \\
\text { any time. }\end{array}$ \\
\hline $\begin{array}{c}\text { Measured } \\
\text { service }\end{array}$ & $\begin{array}{l}\text { Cloud resource usage can be measured, controlled, and } \\
\text { reported providing transparency for both the provider and } \\
\text { the consumer of the utilised service. Cloud services use a } \\
\text { metering capability to control resource usage. }\end{array}$ \\
\hline
\end{tabular}


expansion of the Central Lifecycle Management (LCM), incorporating tracking features and interactive graphical forms. Rudimentary characteristics for integrating compatible services may be offered. A management layer is usually located within cloud architecture to management, which enhance scalable and effective provisioning within the following platforms.

- Cloud Broker Platform: is used for supporting the types of broker activity such as integration, customisation, and aggregation that requires a specified language for description of services in a balanced manner and for definition of the integration mechanism. This originates from the common broker pattern in software design, applied on a cloud environment.

- Cloud Marketplace: originates from broker platforms and it brings customers and providers together. Additionally, description of service for integrated and core services are critical in enhancing technical and functionality quality features. The second aspect that requires facilitation is trust. Notably, apps marketplaces are ubiquitous, thus the marketplace pattern reflects future cloud-specific marketplaces.

\subsection{Resource Management in Cloud Broker}

The support of diverse service consumers by the cloud brokers is based on its ability to integrate resources from more than one cloud provider. The cloud provider receives payment for the resources from the cloud broker, who places premiums on the charges to the consumers. Therefore, there is the maximization of profits among the cloud providers and the cloud brokers. Essentially, the cloud provider targets the satisfaction of the SLA for the hosted services through the utilization of minimal hardware resources amount that is received from the cloud broker [6]. On the other hand, the cloud broker targets the improvement of the profits through the leasing of optimal percentage for the resources of computing provided to the consumers. Additionally, the environment of computing has unpredictable differences leading to an excess arrival rate of web request more than the expected value. Thus, it becomes necessary for the cloud broker to be requested for additional resources by the consumers. Therefore, it is important for the consumers to negotiate with the cloud provider for the resources by relying on pricing schemes that support in maintaining the SLA as well as minimize the cloud resources costs [6]. Furthermore, additional resources may be required by the multiple consumers via the cloud broker. Thus, it may lead to competition over the limited resources by the cloud providers.

The majority of the researchers now focus their attention towards the solutions addressing resource management challenges between the service users and the cloud providers. Mostly, the approaches fail to address the problems of management of the resources within the framework of the enterprise. The reliance on the cloud brokers has the advantage of the possibility for integration of many cloud providers; thus, require exposure to the requirements of orchestration. The recent literature has indicated that the efficient operation and management of the cloud computing environment is essential because the framework for federated cloud computing has increased in demand. Therefore, the cloud brokers provide the best hope for dealing with the federated cloud environment complexities. In that regard, it emphasizes the need for efficient management and allocation of the resources in the model of relying 


\section{Cloud Service Brokerage Roles}

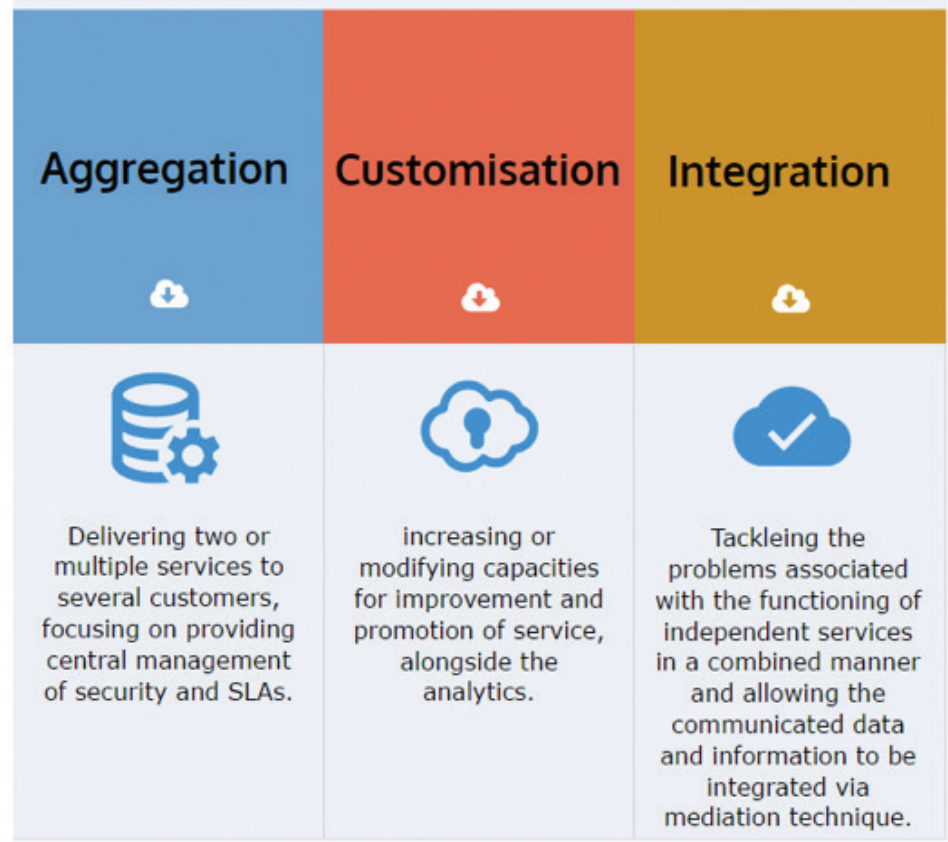

Figure 2 Cloud Service Brokerage Roles

on cloud brokers [17].

\section{Literature Review}

\subsection{Resources Allocation}

Due to the increasing demands of users for provisioning cloud services, the cloud infrastructure faces the challenge of efficient and accurate allocation of resources to the user demands in order to satisfy the agreed SLA. The work of various research and academia communities suggested the utilization of diverse resource allocation techniques that help in maintaining the SLA. The development of a dynamic method for resource allocation entails the considerations for the SLA between the Softwareas-a-Service (SaaS) provider and the user during the resource allocation [6]. The SaaS (Software as a service) provider deal with the heterogeneity of the VMs (Virtual Machines), map the requests of the customers to parameters of infrastructure levels, and manage the changes in requests of customers. The technique considers the QoS (Quality of Service) for the customer including the response time and the parameters of infrastructure levels. However, the major challenge for the laaS cloud provider may be in the evaluation of the SLAs between the SaaS providers and the user especially with the large number of SaaS providers. The resource allocation technique that is based on priority is another approach to allocating the resources as presented in [18], [19]. 
These approaches are categorized into the resource priority based and the user priority based. Essentially, the approaches have bias considerations for the single service provider that support in solving the problem of load balancing. The introduction of the resource allocation based on a neural network in [20] focuses on the maximization of the use of the resource through the strategy of allocating resources offered by genetic algorithm. The technique focuses on the resource-abundant systems. In that regard, the users do not compete for the resources.

The cloud resource allocation is the recent focus of the researchers who apply schemes of auctioning to the SaaS providers. Therefore, the requests of the SaaS providers are accepted by the laaS cloud providers who auction the cloud resources to allocate the highest bidder. The majority of the researchers are focusing on the approaches of game theory studies to solve the complexities of allocating resources in evolutionary and dynamic environments. The mechanism of allocating the resources based on the game theory has been focused on in cloud computing for addressing the problem of optimization of resource allocation [21]. However, according to the recent surveys, the techniques fail to consider the parameters such as resource reliability, execution efficiency, service deadline, resource availability, and fairness. Moreover, A combinatorial auction-based mechanism is investigated for the pricing and allocation of the VMs in the platforms of cloud computing. The approach relies on three schemes including the greedy scheme, linear programming, and the fixed price scheme. The approach has the weakness of only considering the maximization of the user gains while limiting the allocation of the VM types to a value that is pre-determined [22].

\subsection{Workflow scheduling}

A study proposed by Stefansson et al. [23] presents an extensive production scheduling. The researchers offer an evaluation among two mathematical formalisms for a pharmaceutical production scheduling as a MILP (mixed integer linear programming), with one employing a discrete-timeline while the other employs a continuous-timeline. The researchers came to a conclusion that utilisation of a continuous-timeline represents the real-world decision problem and the solutions are more accurate, while the other formula discrete-timeline increases the simplicity of the structure model hence addition of complex constraints can be achieved easily. Another study conducted by Floudas et al. [24] included an outline of the general progression in MILP for the scheduling in chemical processing schemes. The researchers argue that solution precision would escalate by minimizing the time interval (slots) duration, with solver running time being higher besides that of the restraints and variables. Regardless the fact that these studies offer literature on time discretion of scheduling problems, the cloud computing has not been considered in any of them.

As described in Tordsson et al [25], the researchers present an approach based on ILP formulation as a way of optimising the placement of independent tasks in multicloud providers respective of user-specified criteria. Furthermore, the authors employ a static cloud brokering technique besides using "IBM CPLEX solver 1" to evaluate simulations, whereby execution time is increase significantly as the number of tasks goes up. Another study by Van den Bossche et al. [26] produced an independent task-scheduling with ILP formulation as a way of reducing the expense of external 
provisioning in hybrid clouds. For the purpose of quickly acquiring feasible options, the authors decided to have the scheduling problem simulated to decrease tasks quantity to $(\approx 20)$. Regardless the fact that this paper offers a study in scheduling problems in cloud computing settings, none of them consider the implementation of workflows. Furthermore, the literature equally outlines limitations related to the number of tasks against the scheduler execution times.

\subsection{Service assurance and optimization}

The domain of adaptive SBS (service-based systems) [27] deals with the establishment of approaches for monitoring and employing SBS, which share similar characteristics and problems with cloud services. Hence, approaches from this field can be employed for the framework of cloud service brokerage, primarily needing the intermediary to monitor heterogeneous sources' data to determine systems as well as comparing those symptoms with further knowledge to predict or determine failures. An extensive high-tech analysis can be found in [28], where approaches used in adapting or monitoring SBS are described, besides methods for multi- and cross-layer monitoring and adaptation [35], plus relevant work on failure prevention in SBS. The survey addresses several challenges in relevant to cloud service recovery and failure prevention that are not covered in the existing studies; inclusive of (a) the techniques that can be engaged to identify failures of cloud services and what data should be gathered by a broker, (b) the approach to be engaged by the broker to avoid overwhelming situations while monitoring of the different metrics regarding a wide range of cloud services, which are offered by many cloud providers and (c) the appropriate prediction approach to be used by cloud service broker in identifying an impending failure, founded on a metric. Furthermore, a number of additional architectural concerns linked with extensibility, dynamicity and flexibility should be considered.

Authors in [29] implemented a service recommender framework employing VM (Virtual Machine) and Networks QoS technique assisting user's decisions regarding cloud computing optimization, addressing only laas issues. In addition, a study in [30] engaged the use of the so-called service optimizer (SO) used in managing dynamics SLAs. It has been confirmed that the existing work has highly focused on the optimization methodologies instead of the optimization process all together. Existing literature, such as [31], highly focuses on laaS layer and doesn't reflect on the variety of changing conditions that may happen in a cloud service ecosystem. Furthermore, prevailing optimization approaches use only measurable (quantitative) metrics in the evaluation of service characteristics [32].

\subsection{Multi-clouds brokerage threats}

The threats that cloud computing encounter are similar to most corporate networks. The increased number of collaborative parties in a multi-clouds environment such as cloud broker leads to an increased number of connections via networked systems and thus increases the system exposure to threats [33]. The major vulnerabilities in cloud computing brokerage system are therefore as a direct result of the ubiquitous nature of using cloud-based networked systems, as follows: 
- The system is now able to deliver and integrate services from any location or vendor.

- Authorised users should be able to interact with the services from anywhere at any time.

As a result, there are a number of networking threats that should be considered as relevant here: Insider attacks, Equipment failures, End-to-end issues, Data loss or corruption, DDoS attacks, Cyber threats and hacking attacks, Espionage

These threats may be innocent or malicious; however, the fundamental issue is that the most of Critical Infrastructure is denied access to its data or services or that its confidential data may fall into the hands of another party. Thus, these represent the core requirements that must be met in our work.

\section{Discussion}

Utilising services and resources from multi-clouds is forced by consumers who focus on further enhancing the business worth by purchasing services and resources that best suit specific business requirements. An enhanced level of decision support is needed to gain values from adopting laaS, particularly in consideration of multi-clouds providers' resources. Specially, the complexity is linked with locating an appropriate set of resources corresponding to specific application-driven resource requirements and restraints while maximizing the value respective of cost and quality of service. Due to the growing number of cloud providers offering various services and resources with diverse aspects for different prices, even use of varied pricing systems, complexity of the process related to decision making, become more if conflicting objectives are considered. Furthermore, there is the need for consumers to respond on variations in prices and features occurring in over time. As reviewed above, the following table 3 and Fig. 3 and shows that the investigated researches in utilising services and resources in multi-clouds, do not support the decision making process to enable customers of purchasing services and resources that best suit their requirements.

As shown in table 4 and stated in [34], either dynamic or static technique can

TABLE 3 investigated researches in utilising services and resources in multi-clouds.

\begin{tabular}{|l|c|c|c|c|}
\hline & $\begin{array}{c}\text { Decision } \\
\text { support }\end{array}$ & Cost & $\begin{array}{c}\text { Quality of } \\
\text { service }\end{array}$ & Respond time \\
\hline K. Gouda et al. 2013. & $\mathbf{x}$ & $\mathbf{x}$ & $\checkmark$ & $\checkmark$ \\
\hline C.S. Pawar, R.B. Wagh 2012. & $\mathbf{x}$ & $\mathbf{x}$ & $\checkmark$ & $\checkmark$ \\
\hline K. Dinesh et al. 2012. & $\mathbf{x}$ & $\checkmark$ & $\mathbf{x}$ & $\checkmark$ \\
\hline M. Jebalia et al. 2013 & $\mathbf{x}$ & $\checkmark$ & $\mathbf{x}$ & $\checkmark$ \\
\hline S. Zaman, D. Grosu 2013 & $\mathbf{x}$ & $\mathbf{x}$ & $\checkmark$ & $\checkmark$ \\
\hline
\end{tabular}

be employed in tackling the scheduling problem in cloud environments. The static approach is most appropriate in conditions where the cloud provider has high resource availability and accessibility and its provisions (resource configurations and 


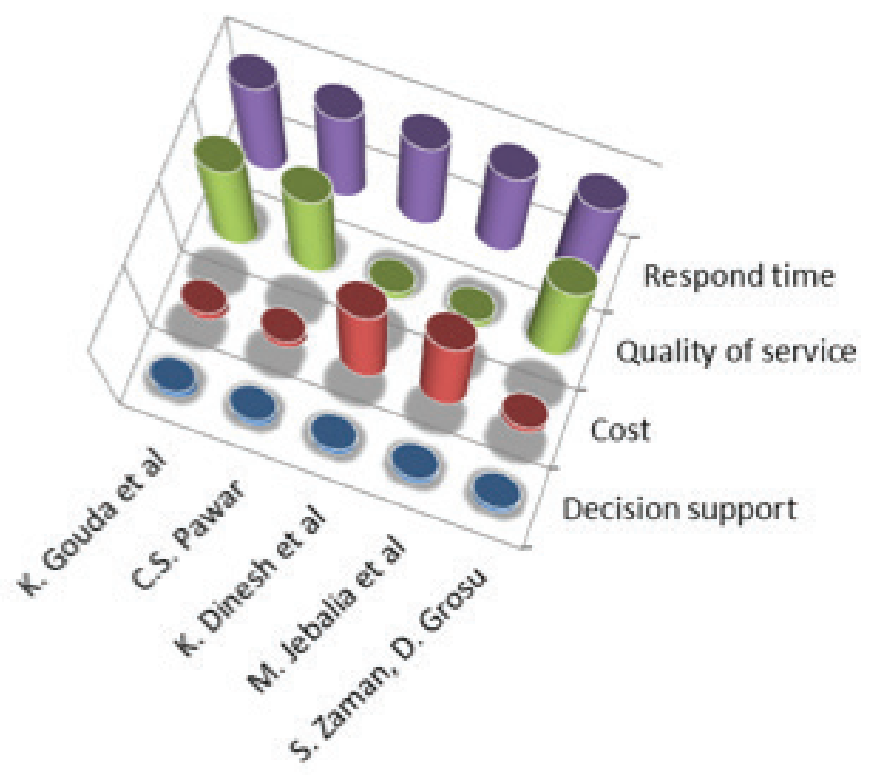

Figure 3. investigated researches in resources allocation.

prices) cannot persistently vary all through the workflow execution lifecycle. Therefore, it is possible to execute workflow scheduling off-line ahead of workflow deployment. Alternatively, the dynamic approach is highly significant in scenarios where uncertainty condition are high, for instance dynamic resource availability, changeable resource costs, variable bandwidth within networks, and so on. In this way, the algorithm in scheduling should be executed online in order to have the cloud environments information updated.

Brokerage abilities for continuous quality assurance and cloud services optimization

TABLE 4. investigated researches in scheduling within cloud environments.

\begin{tabular}{|c|c|c|c|c|c|c|c|}
\hline \multirow{2}{*}{ Scheduling } & \multicolumn{2}{|c|}{ Type } & \multicolumn{2}{c|}{ Rescores } & \multicolumn{2}{c|}{ Execution } & \multirow{2}{*}{$\begin{array}{c}\text { Quality of } \\
\text { service }\end{array}$} \\
\cline { 2 - 7 } & Static & Dynamic & $\begin{array}{c}\text { Avail- } \\
\text { ability }\end{array}$ & $\begin{array}{c}\text { Acces- } \\
\text { sibility }\end{array}$ & $\begin{array}{c}\text { On- } \\
\text { line }\end{array}$ & $\begin{array}{c}\text { Off- } \\
\text { line }\end{array}$ & $\checkmark$ \\
\hline $\begin{array}{c}\text { Stefansson } \\
\text { et al 2011 }\end{array}$ & $\checkmark$ & & $\checkmark$ & $\checkmark$ & & $\checkmark$ \\
\hline $\begin{array}{c}\text { Floudas et } \\
\text { al. 2005 }\end{array}$ & $\checkmark$ & & $\checkmark$ & $\checkmark$ & & $\checkmark$ & $\checkmark$ \\
\hline $\begin{array}{c}\text { J. } \\
\begin{array}{c}\text { Tordsson } \\
\text { et al. 2012. }\end{array}\end{array}$ & $\checkmark$ & $\checkmark$ & $\checkmark$ & & $\checkmark$ & \\
\hline $\begin{array}{c}\text { Van den } \\
\text { Bossche et } \\
\text { al. 2010 }\end{array}$ & $\checkmark$ & & $\checkmark$ & $\checkmark$ & & $\checkmark$ \\
\hline
\end{tabular}


are of enhanced worth to service users, and at the same time, of high complexity for enterprise cloud service brokers to execute. The brokerage structure should presents a range of interfaces promoting interaction among the evolved actors and access to the quality assurance and optimization techniques in an interoperable manner, i.e., as services. In addition, services description has to be considered and exchanged in a platform-neutral manner among different actors involved in brokerage situation. Services description has to reflect different features of a broker service at the operational, business, and technical level. Most of the investigated researches do not consider services description in their platforms as shown in Table 5 and Fig. 4.

TABLE 5 investigated researches in quality assurance and cloud services optimization.

\begin{tabular}{|c|c|c|c|c|}
\hline & Monitoring & $\begin{array}{c}\text { Failure } \\
\text { detection }\end{array}$ & $\begin{array}{c}\text { Quality of } \\
\text { service }\end{array}$ & $\begin{array}{c}\text { Service } \\
\text { description }\end{array}$ \\
\hline V. Yiannis et al 2013. & $\checkmark$ & $\checkmark$ & $\checkmark$ & $\mathbf{x}$ \\
\hline S.-M. Han et al.2009. & $\checkmark$ & $\mathbf{x}$ & $\checkmark$ & $\mathbf{x}$ \\
\hline A. Lawrence et al. 2010. & $\checkmark$ & $\checkmark$ & $\mathbf{x}$ & $\mathbf{x}$ \\
\hline P. Pawluk et al. 2012 & $\checkmark$ & $\mathbf{x}$ & $\mathbf{x}$ & $\mathbf{x}$ \\
\hline S. K. Garg et all 2011 & $\checkmark$ & $\mathbf{x}$ & $\checkmark$ & $\mathbf{x}$ \\
\hline
\end{tabular}

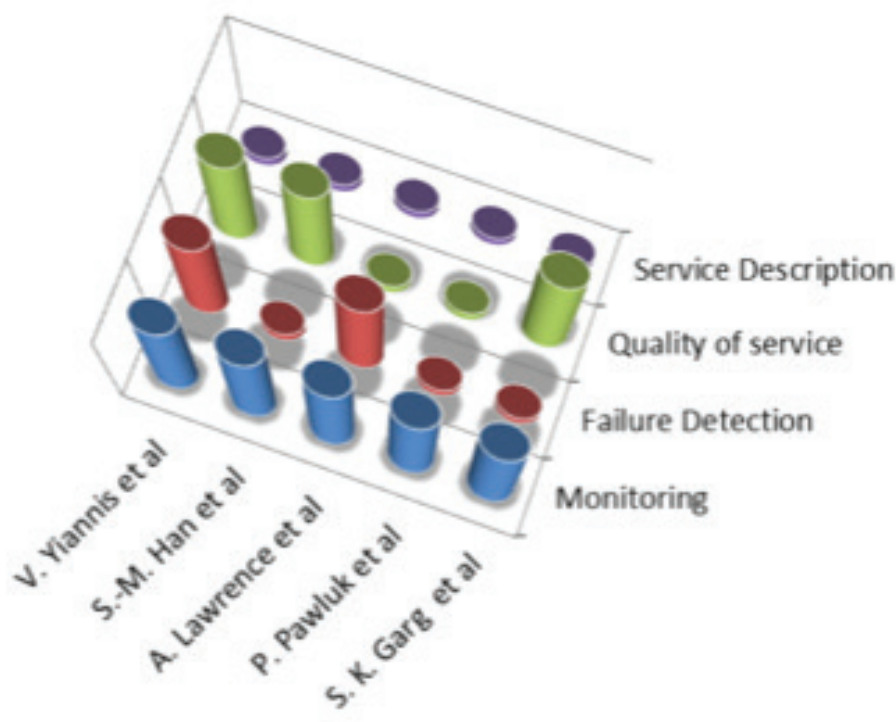

Figure 4 investigated researches in quality assurance and cloud services optimization. 


\section{Conclusion}

Cloud service brokerage represents a new service model in the area of cloud computing, within the domain of cloud computing, focused at helping businesses to handle the complexity of consuming huge numbers of cloud services from diverse sources. Currently, this field is facing a growing number of cloud service mediators that facilitate customization and integration or aggregation brokerage capabilities, however in future; businesses will need much more complicated brokerage services. In this paper we present a Literature on the systems of the cloud brokerage and resource management and the concerns related to them in a multi-cloud environment.

\section{References}

[1] . Furht, B., Escalante, A. (2010). Handbook of cloud computing. New York: Springer.

[2]. Marinescu, D. C. (2013). Cloud Computing: Theory and practice. Boston: Morgan Kaufmann.

[3] . Mehrotra R., Srivastava S., Banicescu I., Abdelwahed S. (2016) Towards an autonomic performance management approach for a cloud broker environment using a decomposition-coordination based methodology, Future Generation Computer Systems, 54, 195-205.

[4] . Roy, A., Dubey, A., Gokhale, L., Dowdy, A. (2011) Capacity planning process for performance assurance of component-based distributed systems, SIGMETRICS Perform. Eval. Rev., 39 (3), 16-17.

[5]. Antonopoulos, N., Gillam, L. (2010). Cloud Computing: Principles, Systems, and Applications. London: Springer.

[6] . Nair, S.K., Porwal, S., Dimitrakos, T., Ferrer, A.J., Tordsson, J., Sharif, T., Sheridan, C., Rajarajan, M., Khan, A.U. (2010) Towards secure cloud bursting, brokerage and aggregation, IEEE $8^{\text {th }}$ European Conference on Web Services, ECOWS, pp. 189-196.

[7]. Hayes, B. (2008) Cloud Computing, Commun. ACM, 51(7), 9-11.

[8] . Obaidat, M. S., Anpalagan, A., Woungang, I., Mouftah, H. T., Kantarci, B. (2013) Handbook of Green Information and Communication Systems, pp. 295-330.

[9] . James, G. (25 September 2012) Power, Pollution and the Internet. The New York Times. Retrieved from: http://www.nytimes.comGholamhosseinian, A., Khalifeh, A. (2012) Cloud Computing and Sustainability: Energy Efficiency Aspects. Master's Thesis in Computer Network Engineering, Technical report, Halmstad University

[10] . Gartner - Cloud Services Brokerage. (2018 January 31). Retrieved from: http:// www.gartner.com/it-glossary/cloud-services-brokerage-csb

[11] . NIST. Cloud Computing Reference Architecture. (2018 January 28). Retrieved from: http://www.nist.gov/customcf/get pdf.cfm?pub id=909505. 2011

[12] . ForresterResearch.CloudBrokersWillReshapeTheCloud.(2018). Retrievedfrom: http://www.cordys.com/ufc/file2/cordyscms, sites/download/09b57cd3eb6474f1fda $1 \mathrm{cfd62ddf094d/pu/}$

[13] .Zhang, L., Fowley, F., Pahl, C. (2014). A template description framework for services as a utility for cloud brokerage. Proceedings of the International Conference on Cloud Computing and Service Science, Fehling. Available at: http://doras.dcu. 
ie/19796/

[14] .Sun, L., Dong, H., Ashraf, J. (2012) Survey of Service Description Languages and Their Issues in Cloud Computing. Proceedings of the Eighth International Conference on Semantics, Knowledge and Grids. pp. 128-135. Available at: http:// ieeexplore.ieee.org/lpdocs/epic03/wrapper.htm?arnumber $=6391820$

[15] . Fowley, F., Pahl, C., Zhang, L. (2013) A comparison framework and review of service brokerage solutions for cloud architectures. Proceedings of the 1st International Workshop on Cloud Service Brokerage, Berlin, Germany.

[16] . Rogers, O., Cliff, A. (2012) Financial brokerage model for cloud computing, J. Cloud Comput., 1 (1), 1-12.

[17] . Gouda, K., Radhika, T., Akshatha, M. (2013) Priority based resource allocation model for cloud computing, Int. J. Sci. Eng. Technol. Res., 2 (1), 215-219.

[18] . Pawar, C.S., Wagh, R.B. (2012) Priority based dynamic resource allocation in cloud computing, Proceedings of the International Symposium on Cloud and Services Computing, pp. 1-6.

[19] . Dinesh, K., Poornima, G., Kiruthika, K. (2012) Efficient resources allocation for different jobs in cloud, Int. J. Comput. Appl., 56 (10), 30-35.

[20] . Jebalia, M., Letaïfa, A.B., Hamdi, M., Tabbane, S. (2013) A comparative study on game theoretic approaches for resource allocation in cloud computing architectures, IEEE 22nd International Workshop on Enabling Technologies: Infrastructure for Collaborative Enterprises, pp. 336-341

[21] . Zaman, S., Grosu, D. (2013) Combinatorial auction-based allocation of virtual machine instances in clouds, J. Parallel Distrib. Comput., 73(4), 495-508.

[22] . Stefansson, H., Sigmarsdottir, S., Jensson, P., Shah, N. (2011) Discrete and continuous time representations and mathematical models for large production scheduling problems: A case study from the pharmaceutical industry, European Journal of Operational Research, 215(2), 383 - 392.

[23]. Floudas, C., Lin, X. (2005) Mixed integer linear programming in process scheduling: Modeling, algorithms, and applications, Annals of Operations Research, 139, 131-162.

[24] . Tordsson, J., Montero, R. S., Moreno-Vozmediano, R., Llorente, I. M. Cloud brokering mechanisms for optimized placement of virtual machines across multiple providers, Journal of Future Generation Computer Systems, 28 (2), 358-367.

[25] . Van den Bossche, R., Vanmechelen, K., Broeckhove, J. (2010) Cost-optimal scheduling in hybrid laaS clouds for deadline constrained workload, Proceedings of the $3^{\text {rd }}$ International Conference on Cloud Computing, pp. 228 - 235.

[26] . Papazoglou, M., Pohl, K., Parkin, M., Metzger, A. (2010) Service research challenges and solutions for the future internet: S-cube - towards engineering, managing and adapting service-based systems. Berlin: Springer-Verlag.

[27]. Han, S.M., Hassan, M. M., Yoon, C.W., Huh, E.N. (2009) Efficient service recommendation system for cloud computing market, Proceedings of the $2^{\text {nd }}$ Int. Conf. on Interaction Sciences: IT, Culture and Human, pp. 839-845.

[28] . Lawrence, A., Djemame, K., Waldrich, O., Ziegler, W., Zsigri, C. (2010) Using service level agreements for optimising cloud infrastructure services, Towards a Service-Based Internet. ServiceWave, Ghent, Beelgium, pp. 38-49. 
[29] .Pawluk, P., Simmons, B., Smit, M., Litoiu, M., Mankovski, S. (2012) Introducing STRATOS: A cloud broker service, Proceedings of the $5^{\text {th }}$ International Conference on Cloud Computing, pp. 891-898.

[30] . Garg, S. K., Versteeg, S., Buyya, R. (2011) SMICloud: a framework for comparing and ranking cloud services, Proceedings of the $4^{\text {th }}$ IEEE International Conference on Utility and Cloud Computing, pp. 210-218.

[31] . Baker, T., Mackay, M., Shaheed, A., Aldawsari, B. (2015) Security-Oriented Cloud Platform for SOA-Based SCADA, Proceedings of the $15^{\text {th }}$ IEEE/ACM International Symposium on Cluster, Cloud and Grid Computing, pp. 961-970.

[32] . Bittencourt, L. F., Madeira, E. R. M., da Fonseca, N. L. S. (2012) Scheduling in hybrid clouds, Communications Magazine, 50(9), pp. $42-47$.

[33].Baker, T., Lamb, D., Taleb-Bendiab, A., Al-Jumeily, D. (2010) Facilitating Semantic Adaptation of Web Services at Runtime Using a Meta-data Layer, Proceedings of the International Conference on Developments in E-Systems Engineering, London, UK. 